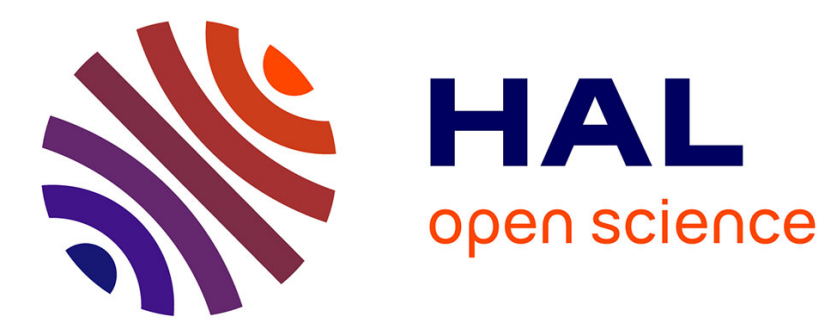

\title{
General fluid-displacement equations for acoustic-gravity waves
}

\author{
Maurice A. Biot
}

\section{To cite this version:}

Maurice A. Biot. General fluid-displacement equations for acoustic-gravity waves. Physics of Fluids, 1963, 6 (5), pp.621-626. 10.1063/1.1706790 . hal-01368706

\section{HAL Id: hal-01368706 https://hal.science/hal-01368706}

Submitted on 21 Sep 2016

HAL is a multi-disciplinary open access archive for the deposit and dissemination of scientific research documents, whether they are published or not. The documents may come from teaching and research institutions in France or abroad, or from public or private research centers.
L'archive ouverte pluridisciplinaire HAL, est destinée au dépôt et à la diffusion de documents scientifiques de niveau recherche, publiés ou non, émanant des établissements d'enseignement et de recherche français ou étrangers, des laboratoires publics ou privés. 


\section{AIP | Physics of}

\section{General Fluid-Displacement Equations for Acoustic-Gravity Waves}

M. A. Biot

Citation: Physics of Fluids 6, 621 (1963); doi: 10.1063/1.1706790

View online: http://dx.doi.org/10.1063/1.1706790

View Table of Contents: http://scitation.aip.org/content/aip/journal/pof1/6/5?ver=pdfcov

Published by the AIP Publishing

\section{Articles you may be interested in}

Acoustic-gravity waves in atmospheric and oceanic waveguides

J. Acoust. Soc. Am. 132, 657 (2012); 10.1121/1.4731213

Two-fluid theory of acoustic-gravity waves in a plasma

Phys. Plasmas 10, 1164 (2003); 10.1063/1.1554742

The dispersion relation for internal acoustic-gravity waves in a baroclinic fluid

Phys. Fluids 13, 1274 (2001); 10.1063/1.1359419

Singular Perturbation Analysis of Acoustic-Gravity Waves

Phys. Fluids 12, 752 (1969); 10.1063/1.1692551

Variational Principles for Acoustic-Gravity Waves

Phys. Fluids 6, 772 (1963); 10.1063/1.1706812

\section{Looking for a specific} instrument?

Easy access to the latest equipment. Shop the Physics Today Buyer's Guide. 


\title{
General Fluid-Displacement Equations for Acoustic-Gravity Waves
}

\author{
M. A. BIot \\ New York, New York \\ (Received 20 September 1962; revised manuscript received 14 January 1963)
}

\begin{abstract}
General equations are derived for the dynamics of a fluid under initial stress in an arbitrary potential field and perturbed from equilibrium. The motion is described in terms of the displacements of the fluid particles from their equilibrium position. A class of equations is obtained which is applicable to large displacements. Complete linearization leads to two types of equations. One type called "unmodified" corresponds to the viewpoint of the theory of elasticity. The "modified" equations representing the other type are expressed in terms of buoyancy forces. The modified equations lead to a conceptually useful analog model for internal gravity waves in a liquid. For a constant gravity field the linear equations are also applicable to large displacements. Classical examples for a constant gravity field are discussed as illustrations.
\end{abstract}

\section{INTRODUCTION}

$\mathrm{T}$ HE theory of wave propagation in a fluid subject to a gravity field has been the object of analytical treatment by many authors in the classical literature.

One may distinguish acoustic-gravity waves represented by the propagation of large-scale disturbances in the atmosphere. This represents the more general case where the propagation is governed by the combined effect of compressibility and gravity.

The particular case of an incompressible fluid corresponds to pure gravity waves occurring in a heavy liquid of nonuniform density. They may be internal gravity waves if they occur in the body of the fluid and are governed by the density gradient or density discontinuity. They include surface waves as a particular case of density discontinuity.

The propagation of gravity waves in heterogeneous liquids has been treated by Love, Burnside, Rayleigh, Lamb. (Classical references will be found in Lamb's treatise, ${ }^{1}$ p. 378.) These studies were initiated already in the late nineteenth century.

General equations for small motion of a gas about a state of equilibrium in any constant field of force have been derived by Lamb (see Lamb's treatise, ${ }^{1}$ p. 554). Many applications have been discussed by Bjerknes $^{2}$ in the context of meteorology. In a recent book Eckart ${ }^{3}$ has treated extensively the problems of hydrodynamies of the ocean and atmosphere. The traditional procedure is founded on Euler's equations of fluid dynamics where the motion is described by a velocity field.

${ }^{1}$ H. Lamb, Hydrodynamics (Cambridge University Press, New York, 1932). [Reprinted by Dover Publications, New York, 1945.]

2 V. Bjerknes et al., Physikalische Hydrodynamik (SpringerVerlag, Berlin, 1933).

${ }^{3}$ C. Eckart, Hydrodynamics of Oceans and Atmospheres (Pergamon Press, Inc., New York, 1960).
The present paper is concerned with a fundamental approach of a different nature. The theory is developed from the standpoint of the fluid displacement instead of the velocity field. In this formulation the coordinates of a given fluid particle are considered as functions of the initial coordinates and the time. The equations constitute a special case of the theory of elasticity and elastic wave propagation under initial stress derived by the writer in 1940. ${ }^{4}$ By inserting a zero value for the shear modulus the equations reduce to that of a fluid under initial stress. This reduction is trivial and immediate.

While the results obtained from the viewpoint of the elasticity theory are completely general there is need for a more complete treatment and discussion in the case of a fluid.

Our purpose here is to present such a treatment in rigorous and systematic form for a fluid in a nonuniform body force field.

General equations for the motion of a fluid perturbed from equilibrium are derived in Sec. 2 . A class of equations is obtained which is applicable for large displacements provided the displacement gradients remain small.

By further linearization with respect to the displacements it is possible to derive two essentially different types of equations as shown in Sec. 3. In what we have called the "unmodified form" the equations embody the viewpoint of the theory of elasticity. Mathematically equivalent "modified equations" can be derived which emphasize the viewpoint of the mechanics of fluids by introducing the buoyancy forces. The modified equations are closely related to Euler's equations.

${ }^{4}$ M. A. Biot, J. Appl. Phys. 11, 522 (1940). 
The case of an incompressible fluid is treated in Sec. 4. It includes internal gravity waves in a nonhomogeneous liquid. A conceptually very useful analog model is derived for this case. It is shown that the motion is the same as in a fluid free of gravity provided we add elastic forces, proportional to the displacement, distributed inside the fluid and at the free surface.

Particular forms of these equations for the case of a constant gravity field are discussed in Sec. 5 leading to classical results.

Use of the displacement field equations instead of the Eulerian description has many advantages. It provides a clearer and more general physical description, and leads immediately to variational principles. These principles will be derived in the next paper. ${ }^{5}$

The displacement-field equations also provide a link with the theory of elasticity and a unified theory applicable to both fluids and solids. This is particularly useful in the analysis of composite fluid-solid media. The equations considered as a particular case of the theory of elasticity will be derived in a third paper. ${ }^{6}$

\section{DYNAMICS OF A FLUID CONTINUUM UNDER INITIAL STRESS}

Consider a fluid in a state of equilibrium under a body force field. This state of equilibrium will be referred to as the "initial state." The force field acting on the fluid per unit mass is represented by its components

$$
X_{i}=X_{i}(x) .
$$

They are functions of the coordinates $x, y, z$ designated in abbreviated form as $x$. The initial stress in the fluid is an isotropic stress field designated as

$$
S=S(x)
$$

represented by the initial fluid pressure changed in sign. The initial mass density of the fluid is a function of the coordinates

$$
\rho=\rho(x) .
$$

No relation is assumed $a$ priori between $\rho$ and $S$ with the implication that the fluid may be heterogeneous. The distribution of heterogeneity may be chosen arbitrarily. The only requirement of this initial state is that the fluid be in mechanical equilibrium, hence that the following equation be satisfied:

$$
\partial S / \partial x_{i}+\rho(x) X(x)=0 .
$$

This equilibrium condition implies a relation between the body force field and the density. In vector

\footnotetext{
6 M. A. Biot, Phys. Fluids (to be published).

${ }^{6}$ M. A. Biot, Phys. Fluids (to be published).
}

notation this relation is written

$$
\operatorname{curl}(\rho \mathbf{X})=0 .
$$

Let the fluid be disturbed from equilibrium by a small perturbation. A mass particle originally at the point $x, y, z$ is displaced to a point of coordinates $\xi, \eta, \zeta$. We write

$\xi=x+u, \quad \eta=y+v, \quad \zeta=z+w$.

The components of the displacement vector of the particle are $u, v, w$. We shall designate the coordinates $\xi, \eta, \zeta$ by $\xi_{i}$ and the components $u, v, w$ by $u_{i}$. Equations (2.6) then assume the abbreviated form

$$
\xi_{i}=x_{i}+u_{i} .
$$

Consider now the dynamical equations for the fluid in this perturbed condition. We shall assume here that in this perturbed motion the fluid particle behaves as a frictionless fluid. Hence the stress in the perturbed fluid remains isotropic. A fluid particle originally at point $x_{i}$ has moved to the point $\xi_{i}$. The isotropic stress on the displaced particle has now become $\sigma$, its density $\rho^{\prime}$, and its acceleration is $a_{i}$. The dynamical equations for the motion of this particle are

$$
\partial \sigma / \partial \xi_{i}+\rho^{\prime}(\xi) X_{i}(\xi)=\rho^{\prime}(\xi) a_{i} .
$$

These equations are expressed in terms of the coordinates $\xi_{i}$ as independent variables. The problem is to transform these equations so that the original coordinates $x_{i}$ become the independent variables. An equivalent form of Eqs. (2.8) is

$$
\left(\partial \sigma / \partial x_{j}\right)\left(\partial x_{j} / \partial \xi_{i}\right)+\rho^{\prime} X_{i}=\rho^{\prime} a_{i} .
$$

The usual summation rule is assumed in the notation. The partial derivatives $\partial x_{i} / \partial \xi_{i}$ are easily expressed in terms of the inverse derivatives by the standard procedure. We write the total differentials

$$
d \xi_{i}=\left(\partial \xi_{i} / \partial x_{i}\right) d x_{i}
$$

and solve this system for $d x_{i}$,

$$
d x_{i}=(1 / J) M_{i i} d \xi_{i} ;
$$

hence

$$
d x_{i} / d \xi_{i}=(1 / J) M_{i j} .
$$

The Jacobian of the transformation (2.10) is

$$
J=\left|\begin{array}{lll}
\frac{\partial \xi}{\partial x} & \frac{\partial \xi}{\partial y} & \frac{\partial \xi}{\partial z} \\
\frac{\partial \eta}{\partial x} & \frac{\partial \eta}{\partial y} & \frac{\partial \eta}{\partial z} \\
\frac{\partial \zeta}{\partial x} & \frac{\partial \zeta}{\partial y} & \frac{\partial \zeta}{\partial z}
\end{array}\right| .
$$


The cofactors of this determinant are $M_{i i}$. They are the partial Jacobians with suitable signs. They contain terms which are linear and quadratic in the displacement gradients $\partial u_{i} / \partial x_{i}$. In evaluating $M_{i j}$ we shall introduce the assumption that the displacement gradients are small quantities such that their squares and products may be neglected. This is equivalent to the condition that the strain and rotations are small. However, no such restriction is imposed on the magnitude of the displacement $u_{i}$. Under these conditions we may write

$$
M_{i j}=(1+e) \delta_{i i}-\partial u_{j} / \partial x_{i} .
$$

In this expression $e$ is the dilatation

$$
e=\frac{\partial u}{\partial x}+\frac{\partial v}{\partial y}+\frac{\partial w}{\partial z}=\frac{\partial u_{i}}{\partial x_{i}}
$$

and $\delta_{i j}$ is the Kronecker symbol

$$
\delta_{i j}= \begin{cases}1 & i=j, \\ 0 & i \neq j .\end{cases}
$$

Substituting the partial derivatives (2.12) into Eqs. (2.8) and taking into account the law of conservation of mass

$$
\rho=\rho^{\prime} J
$$

we derive

$$
M_{i j} \partial \sigma / \partial x_{i}+\rho(x) X_{i}(\xi)=\rho(x) a_{i} .
$$

The stress $\sigma$ on the particle may be written

$$
\sigma=S+s,
$$

where $-s$ is the pressure increment. If it depends only on the dilatation we write

$$
s=\lambda e
$$

with an incremental bulk modulus $\lambda$. We may choose it to be either the isothermal or adiabatic modulus.

Again by assuming the deformation and rotations to be small we retain only the terms which are linear in the displacements gradients. With this approximation we may write

$M_{i j} \frac{\partial \sigma}{\partial x_{i}}=\frac{\partial s}{\partial x_{i}}+e \frac{\partial S}{\partial x_{i}}-\frac{\partial u_{i}}{\partial x_{i}} \frac{\partial S}{\partial x_{i}}+\frac{\partial S}{\partial x_{i}}$.

When substituting this expression in Eqs. (2.18) we take into account the equilibrium condition (2.4) for the initial stress field and write

$$
\partial S / \partial x_{i}+\rho(x) X_{i}(\xi)=\rho(x) \Delta X_{i}
$$

with

$$
\Delta X_{i}=X_{i}(\xi)-X_{i}(x)
$$

Equation (2.18) becomes

$$
\frac{\partial s}{\partial x_{i}}+e \frac{\partial S}{\partial x_{i}}-\frac{\partial u_{i}}{\partial x_{i}} \frac{\partial S}{\partial x_{i}}+\rho \Delta X_{i}=\rho a_{i} .
$$

The acceleration which appears on the right-hand side is

$$
a_{i}=\partial^{2} u_{i} / \partial t^{2} .
$$

Hence the dynamical equations are

$$
\frac{\partial s}{\partial x_{i}}+e \frac{\partial S}{\partial x_{i}}-\frac{\partial u_{i}}{\partial x_{i}} \frac{\partial S}{\partial x_{i}}+\rho \Delta X_{i}=\rho \frac{\partial^{2} u_{i}}{\partial t^{2}} .
$$

With suitable initial and boundary conditions they determine the displacement field $u_{i}$ as a function of time. The initial mass density $\rho(x)$ and the initial stress $S(x)$ are given functions of the initial coordinates $x_{i}$

Note that Eqs. (2.26) are not necessarily linear in the unknown displacements since $\Delta X_{i}$ are explicit functions of $u_{i}$.

Coriolis acceleration. When the frame of reference is rotating the acceleration $a_{i}$ must include a Coriolis term. This is easily included in the above equations by introducing the antisymmetric matrix

$$
\left[\Omega_{i j}\right]=\left[\begin{array}{rcc}
0 & -\Omega_{z} & \Omega_{y} \\
\Omega_{z} & 0 & -\Omega_{x} \\
-\Omega_{y} & \Omega_{x} & 0
\end{array}\right],
$$

where $\Omega_{x} \Omega_{y} \Omega_{z}$ are the components of the angular velocity of the frame of reference. In this case the acceleration in Eq. (2.24) is replaced by

$$
a_{i}=\partial^{2} u_{i} / \partial t^{2}+2 \Omega_{i j} \partial u_{j} / \partial t .
$$

\section{MODIFIED AND UNMODIFIED EQUATIONS}

The general equations (2.24) may be written in a different form by introducing two assumptions. We assume that the body force is derived from a potential $U$, i.e.,

$$
X_{i}=-\partial U / \partial x_{i} .
$$

In addition we shall linearize $\Delta X_{i}$ by writing

$$
\Delta X_{i}=\frac{\partial X_{i}}{\partial x_{i}} u_{j}=-\frac{\partial^{2} U}{\partial x_{i} \partial x_{i}} u_{j} .
$$

It is well known that the existence of a body force potential implies that in the equilibrium state the surfaces of constant density coincide with equipotential surfaces. This is readily established by combining Eqs. (2.4) and (3.1). We find

$$
\frac{\partial \rho}{\partial x_{i}} \frac{\partial U}{\partial x_{i}}=\frac{\partial \rho}{\partial x_{i}} \frac{\partial U}{\partial x_{i}} .
$$


This equation implies that the body force $X_{i}$ and the density gradient $\partial \rho / \partial x_{i}$ are parallel vectors. Under these assumptions it is possible to derive dynamical equation of two essentially different types as will now be shown.

With the value (3.2) the dynamical equations (2.24) are written

$\frac{\partial s}{\partial x_{i}}+e \frac{\partial S}{\partial x_{i}}-\frac{\partial u_{i}}{\partial x_{i}} \frac{\partial S}{\partial x_{j}}-\rho \frac{\partial^{2} U}{\partial x_{i} \partial x_{i}} u_{i}=\rho a_{i}$.

We shall refer to them as the unmodified equations. In this form the equations are the same as those obtained directly for the linearized equations of elasticity for an initially stressed continuum ${ }^{4}$ as will be shown in another paper. ${ }^{6}$

Another equivalent form of Eqs. (3.4) is obtained as follows. We put

$$
s=s^{\prime}+\rho u_{j} \partial U / \partial x_{i} .
$$

Substitution in Eqs. (3.4) taking into account Eqs. (2.4) and (3.1) yields

$$
\frac{\partial s^{\prime}}{\partial x_{i}}-\rho e X_{i}-u_{i} X_{j} \frac{\partial \rho}{\partial x_{i}}=\rho a_{i} .
$$

We shall refer to these equations as the modified equations. By using Eq. (3.1) the variable $s^{\prime}$ is also written

$$
s^{\prime}=s+\rho u_{j} X_{i} .
$$

The interest of the modified form lies in their physical interpretation and intuitive value.

To show this we rewrite the value (3.7) of $s^{\prime}$ by taking into account the equilibrium condition (2.4)

$$
s^{\prime}=s-u_{i} \partial S / \partial x_{i} .
$$

This expression shows that $s^{\prime}$ is the increment of stress at the fixed point $x_{i}$.

On the other hand, let us look at the terms containing the body force in Eq. (3.6). Taking into account relations (3.3) we write

$$
\rho e X_{i}+u_{i} X_{i} \frac{\partial \rho}{\partial x_{i}}=X_{i} \frac{\partial}{\partial x_{i}}\left(\rho u_{j}\right) .
$$

These terms represent the buoyancy force on the fluid in a fixed volume of space. It is directed normally to the equipotential surface. To the first order this buoyancy force and the acceleration $a_{i}$ may be considered as values at the fixed point $x_{i}$.

With this interpretation Eq. (3.6) becomes intuitively self-evident as expressing Newton's law for a fluid particle at the fixed point.

Attention is called to an important property for the case of a constant gravity field. In this case
$\Delta X_{i}$ drops out and linearization is not required. Hence for a constant gravity field the modified equations (3.6) are equivalent to Eqs. (2.24) and are therefore applicable to large displacements.

Relation to Euler's equations of fluid dynamics. Using relation (3.9) the modified equations (3.6) become

$$
\frac{\partial s^{\prime}}{\partial x_{i}}-X_{i} \frac{\partial}{\partial x_{i}}\left(\rho u_{j}\right)=\rho a_{i} .
$$

This result is closely related to the equations derived from fluid dynamics. Euler's equations are

$$
\rho\left(\frac{\partial v_{i}}{\partial t}+v_{i} \frac{\partial v_{i}}{\partial x_{i}}\right)=-\frac{\partial p}{\partial x_{i}}+\rho X_{i},
$$

where $v_{i}$ and $p$ denote the fluid velocity and pressure at the fixed point $x_{i}$. Taking the time derivative of Eq. (3.11) neglecting higher order terms yields

$$
\rho \frac{\partial^{2} v_{i}}{\partial t^{2}}=-\frac{\partial}{\partial x_{i}}\left(\frac{\partial p}{\partial t}\right)+X_{i} \frac{\partial \rho}{\partial t} .
$$

The equation of conservation of mass is

$$
\partial \rho / \partial t+\partial\left(\rho v_{i}\right) / \partial x_{i}=0 .
$$

Hence, eliminating $\partial \rho / \partial t$ between the last two equations we obtain

$$
\rho \frac{\partial^{2} v_{i}}{\partial t^{2}}=-\frac{\partial}{\partial x_{i}}\left(\frac{\partial p}{\partial t}\right)-X_{i} \frac{\partial}{\partial x_{i}}\left(\rho v_{i}\right) .
$$

This result is identical in form with Eq. (3.10). The velocity $v_{i}$ replaces the displacement $u_{i}$ and $-\partial p / \partial t$ replaces $s^{\prime}$. Dividing Eq. (3.10) by an infinitesimal time interval $\Delta t$ yields Euler's equations (3.14) in the limit.

\section{ANALOG MODEL FOR INTERNAL GRAVITY WAVES IN A LIQUID}

In an incompressible liquid we put

$$
e=0
$$

in the modified equations (3.6). They are simplified to

$$
\frac{\partial s^{\prime}}{\partial x_{i}}-u_{i} X_{i} \frac{\partial \rho}{\partial x_{i}}=\rho a_{i} .
$$

Equations (4.1) and (4.2) are four equations for $s^{\prime}$ and the three displacement components $u_{i}$.

They are identical with the dynamical equations for a liquid initially stress free with distributed body forces acting on the fluid particles proportionally to their displacement from equilibrium.

This new physical system may be considered as an analog model for the actual fluid under initial stress.

The stress in the model is $s^{\prime}$. It is different from 
the stress in the actual fluid. The incremental stress $s$ in the actual fluid is related to the model stress $s^{\prime}$ by Eq. (3.7),

$$
s=s^{\prime}-\rho u_{i} X_{i} .
$$

This shows that a free surface in the actual fluid is not a free surface in the model. By putting $s=0$ in Eq. (4.3) the following boundary condition in the model is obtained:

$$
s^{\prime}=\rho u_{i} X_{i} .
$$

Since $X_{i}$ is normal to the free surface we may write

$$
s^{\prime}=\rho u_{n} X,
$$

where $u_{n}$ is the normal displacement and $X$ the normal component of the body force.

Hence at the surface of the model we must apply a normal force $s^{\prime}$ per unit area, proportional to the boundary normal displacement. If the body force is directed inward at the surface this force acts in opposition to the displacement as an elastic restoring force. In that case it is stabilizing. In the opposite case it acts as a negative elastic force and is destabilizing. This corresponds to the so-called "Taylor instability."

The body force of the model can similarly be looked upon as a positive or negative elastic force applied to the fluid particles per unit volume. We may write

$$
-u_{j} X_{i} \partial \rho / \partial x_{i}=-\left(u_{n} X \partial \rho / \partial n\right) n_{i}
$$

where $n_{i}$ is the unit normal to the equipotential surface with $\partial \rho / \partial n$ equal to the normal derivative of the density along the same direction. We see that if $X$ and $\partial \rho / \partial n$ are of the same sign, expression (4.6) represents an elastic restoring force. Hence it is stabilizing. If $X$ and $\partial \rho / \partial n$ are of opposite sign the force is destabilizing.

If there is a surface of discontinuity for the density we may think of it as a thin layer through which the value of the density varies rapidly from $\rho_{1}$ to $\rho_{2}$. By integration across the thickness expression (4.6) becomes a force per unit area $-u_{n} X\left(\rho_{2}-\rho_{1}\right) n_{i}$ applied to the surface of discontinuity. This includes the boundary surface which may be considered as a density discontinuity by putting $\rho_{2}=0$.

Hence if the liquid is composed of layers each of which is of constant density, the analog model is obtained by applying forces to the discontinuity surfaces including the free surface.

\section{CONSTANT GRAVITY FIELD}

With a vertical $z$ axis positive upward a constant gravity field of acceleration $g$ is represented by the components

$$
X_{i}=(\mathbf{0}, \mathbf{0},-g) .
$$

The unmodified equations (3.4) become

$$
\begin{aligned}
\frac{\partial s}{\partial x}-\rho g \frac{\partial w}{\partial x} & =\rho \frac{\partial^{2} u}{\partial t^{2},} \\
\frac{\partial s}{\partial y}-\rho g \frac{\partial w}{\partial y} & =\rho \frac{\partial^{2} v}{\partial t^{2}}, \\
\frac{\partial s}{\partial z}+\rho g e-\rho g \frac{\partial w}{\partial z} & =\rho \frac{\partial^{2} w}{\partial t^{2}} .
\end{aligned}
$$

Equations of this type were also proposed earlier by the writer. ${ }^{4}$ To these we must add Eq. (2.20) for $s$. By the change of variable

$$
s^{\prime}=s-\rho g w
$$

taking into account that $\rho$ is a function of $z$ only equations (5.2) become

$$
\begin{aligned}
\frac{\partial s^{\prime}}{\partial x} & =\rho \frac{\partial^{2} u}{\partial t^{2}}, \\
\frac{\partial s^{\prime}}{\partial y} & =\rho \frac{\partial^{2} v}{\partial t^{2}} \\
\frac{\partial s^{\prime}}{\partial z}+\rho g e+w g \frac{d \rho}{d z} & =\rho \frac{\partial^{2} w}{\partial t^{2}}
\end{aligned}
$$

They correspond to the modified form (3.6) of the general case. As already pointed out, Eqs. (5.2) and (5.4) are equivalent for the case of a constant gravity field and both applicable to large displacements. Equations equivalent to Eqs. (5.4) but slightly different in form were also proposed by Eliassen and Kleinschmidt ${ }^{7}$ using the same displacement field.

For a liquid we put $e=0$. Hence

$$
\begin{aligned}
\frac{\partial s^{\prime}}{\partial x} & =\rho \frac{\partial^{2} u}{\partial t^{2}}, \\
\frac{\partial s^{\prime}}{\partial y} & =\rho \frac{\partial^{2} v}{\partial t^{2}}, \\
\frac{\partial s^{\prime}}{\partial z}+w g \frac{d \rho}{d z} & =\rho \frac{\partial^{2} w}{\partial t^{2}} .
\end{aligned}
$$

These equations represent the analog model. If $d \rho / d z$ is negative the body force in the model is a restoring elastic force $w g(d \rho / d z)$ proportional to the vertical displacement. At the surface of the model a restoring force is also applied. Its value per unit area is

$$
s^{\prime}=-\rho g w .
$$

${ }^{7}$ A. Eliassen and E. Kleinschmitt, in Handbuch der Physik, edited by S. Flügge (Springer-Verlag, Berlin, 1957), Vol. 48, p. 52 . 
As an illustration we apply Eqs. (5.5) to the classical case of two dimensional motion in an ocean of constant depth $h$. We put

$$
u=\frac{\partial \psi}{\partial z}, \quad v=0, \quad w=-\frac{\partial \psi}{\partial x},
$$

and

$$
\psi=\exp (i k x-i \omega t) f(z) .
$$

This solution satisfies the condition $e=0$ of incompressibility. Substitution of the values (5.7) into Eqs. (5.5) yields two equations for $s^{\prime}$ and $f$. By elimination of $s^{\prime}$ we are left with a Sturm-Lionville equation for $f$.

$$
\frac{d}{d z}\left(\rho \frac{d f}{d z}\right)+\frac{\rho}{V^{2}}\left(\omega_{c}^{2}-\omega^{2}\right) f=0 .
$$

We have put

$$
\omega_{c}^{2}=-g \frac{1}{\rho} \frac{d \rho}{d z}, \quad \frac{\omega}{k}=V .
$$

The parameter $V$ is the phase velocity along $x$. The boundary condition at the surface is derived from equations (5.5) and (5.6) and is written

$$
\frac{g}{V^{2}} f=\frac{d f}{d z} .
$$

The other boundary condition at the rigid bottom is $f=0$.

\section{ACKNOWLEDGMENT}

This work was supported by the Air Force Office of Scientific Research under contract No. AF$49(638)-837$.

\title{
Flow of a Non-Newtonian Fluid between Rotating Cylinders with Suction and Injection
}

\author{
J. N. KAPUR \\ Indian Institute of Technology, Kanpur, India \\ AND \\ Shashi Goel \\ University of Delhi, Delhi, India
}

(Received 19 July 1961; revised manuscript received 27 November 1962)

\begin{abstract}
The steady flow of a certain non-Newtonian fluid in an annulus between two coaxial cylinders rotating with uniform angular velocities about the common axis is studied when there is suction at one wall and injection at the other. The stress matrix $T$ for the non-Newtonian fluid is given by $T=-p I+\alpha_{1} A_{1}+\alpha_{2} A_{2}$, where $p$ is the pressure, $I$ is the unit matrix, $\alpha_{1}, \alpha_{2}$ are constants and $A_{1}$, $A_{2}$ are kinematic matrices. It is found that in the case of no suction and injection, the velocity field is not affected by the presence of the non-Newtonian term $\alpha_{2} A_{2}$, though the pressure field is affected. On the other hand, if there is suction and injection, however small, the non-Newtonian term affects the velocity field and the nature of this effects is investigated for sufficiently small suction and injection velocities.
\end{abstract}

\section{INTRODUCTION}

$\mathrm{T}$ HE steady flow of a Newtonian fluid in the annulus between two coaxial cylinders rotating with uniform angular velocities about the common axis when there is suction at one wall and injection at the other has been studied by Kapur and Mallick. ${ }^{1}$ In the present paper we study the same problem for a non-Newtonian fluid whose constitutive equation is

$$
T=-p I+\alpha_{1} A_{1}+\alpha_{2} A_{2},
$$

\footnotetext{
${ }^{1}$ J. N. Kapur and S. C. Mallick, Proceedings of the Sixth Congress of Theoretical and Applied Mechanics, India, (1960), Vol. 6, p. 125.
}

where $T$ is the stress matrix $\left\|t_{i J}\right\|, I$ is the unit matrix $\left\|\delta_{i J}\right\|, A_{1}, A_{2}$ are kinematic matrices defined by $^{2}$

$$
\begin{aligned}
& A_{1}=\left\|\left(V_{i, J}+V_{J, i}\right)\right\|=\left\|A_{i J}\right\|=\left\|2 d_{i J}\right\|, \\
& A_{2}=\| \frac{\partial}{\partial t} A_{i J}+V^{i} A_{i J . l} \\
& \quad+A_{m i} V_{J J}^{m}+A_{m J} V_{. i}^{m} \| ;
\end{aligned}
$$

$p$ is a scalar which we identify with pressure and $\alpha_{1}, \alpha_{2}$ are constants. Here $\delta_{i J}$ is the Kronecker set, ${ }^{2}$ J. I. Erickson and Rivlin, J. Ratl. Mech. Anal. 4, 323 (1955). 